\title{
Novel pseudo PMOS ultraviolet photo catalytic oxidation (PP-UVPCO) sensor for air purification
}

\begin{abstract}
The paper presents a performance analysis of Novel CMOS Integrated Pseudo PMOS UVPCO having zero static power dissipation. The main emphasis is on simulation of power and performance analysis along with the comparison with existing devices, which is used for Air Purification. This approach can improve calibration without the use of a high speed digital processor. The conventional devices consume high power and are not firm for long term monitoring. These devices are also suffered from low value of slew rate, high power consumption, and non linear characteristics but in this novel design the device has almost zero static power, less load capacitance on input signals, faster switching, fewer transistors, higher circuit density and the device has better slew rate. This device has a modest architecture, and suitable for Air purification and surface sanitisation.
\end{abstract}

Index Terms-ultra violet photo catalytic oxidation (UVPCO), air purification, slew rate, SPICE, surface sanitization.
Volume 4 Issue 6 - 2018

\author{
Pawan Whig,' Ahmad SN² \\ 'Vivekananada Institute of Professional Studies, GGSIPU, India \\ ${ }^{2}$ Department of electronics and communication engineering, \\ Jamia millia Islamia Central University, India
}

Correspondence: Pawan Whig, Vivekananada Institute of Professional Studies, GGSIPU, India, Tel +91-981।908699, Email pavvan.whig@vips.edu

Received: May 18, 2018| Published: November 30, 2018

\section{Introduction}

Nowaday's air pollution is increasing because of harmful material in earth atmosphere causing deceases, deaths, and damage to crop. These harmful material can be natural origin or man-made. The main source of these harmful materials is power plant and manufacturing factories. The main air pollutant which can have adverse effects on humans and the ecosystem, are sulphur oxide, carbon monoxide, volatile organic compound, chlorofluorocarbons ammonia and toxic metals. These air pollutants can have adverse effect on human health like headache, fatigue, poor memory, respiratory irritation, pneumonia in children, lung cancer, heart disease, eye, and nose and throat irritation.

Hence, there is a need to purify air by using advanced process called Photo Catalytic Oxidation (PCO). In PCO Titanium dioxide is used as a catalyst which cleans the air. ${ }^{1-3}$ As air falls on this catalyst, electron will get energy and released at its surface. Then it will interact with water molecules in the air, as we know that electron having energy which breaks the water molecules into hydroxy1 radicals, hydroxide and hydrogen peroxide. ${ }^{4}$ These hydroxy 1 radicals are more reactive so they attack organic pollutant molecules, breaking them into carbon dioxide and water which are harmless substance. The methods used for photo catalytic oxidation applications includes massive set ups and require ample time for computation and complex calculations. The complete photo catalytic oxidation process is shown in Figure $1^{5,6}$ This process is responsible for purify air up to significant level, to measure that level there is a serious need of sensor which tell the percentage purification of air. Novel CMOS Integrated Pseudo PMOS UVPCO (PP-UVPCO) model having zero static power dissipation is presented in this research studies which gives an air and surface sanitization readings..$^{7-9}$
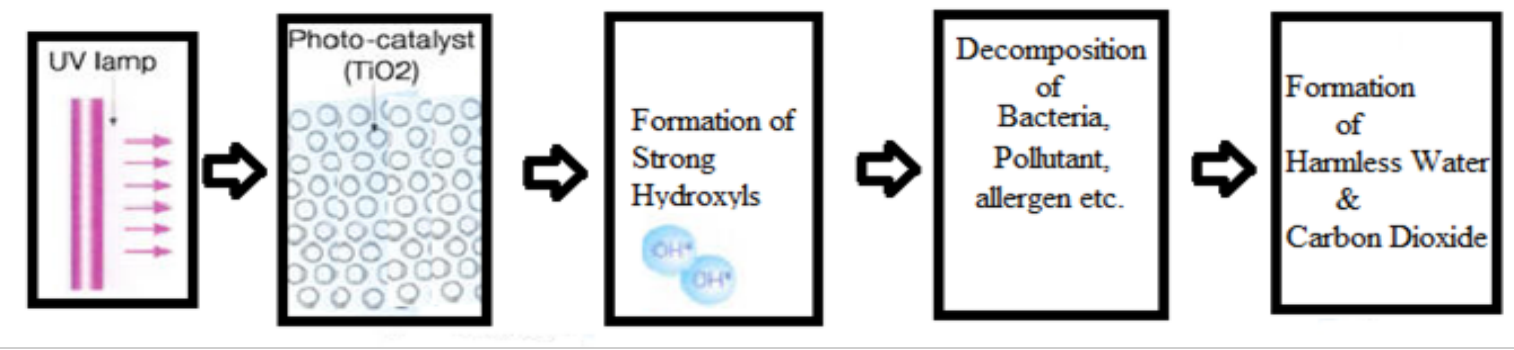

Figure I Process of photo catalytic oxidation.

Now a days due to multipurpose demands i.e all the things on a single chip, complex functions are built. To realize the complex function on the same chip there is growing demand for high density VLSI circuits. One of the practical solutions is to scaling of transistor and Vdd. The major problem which effect is an exponential increase of leakage or static power in deep sub-micron technology. Hence to overcome this problem a portable device for air quality monitoring applications is highly desirable. With the advancement in technology, the channel length of the transistor decreases (i.e Technology) this leads to increase in the transistors density per unit chip area, due to scaling of the device and very large integration of the transistors will lead to increase in temperature and higher power consumption inside the device. ${ }^{10-12}$

The increase in temperature will lead to the increase in the overall cooling cost which further impact the necessary packaging techniques cost. Power plays an important role for selecting any digital device. One of the important parameter on which total power consumption in the modern digital circuits depends is leakage currents. Leakage power contributes to $39.9 \%$ (approx) of the total power consumption in the modern high performance monitoring circuits. ${ }^{13-16}$ Hence, It 
is necessary to work on reduction of leakage current which intern reduces the leakage power and thus useful for a modern digital low power design. The relationship between leakage current and leakage power dissipation is given by (1).

$$
P_{\text {static }}=I_{\text {leak }} * V_{d d}
$$

Where,

$\mathrm{I}_{\text {leak }}$ indicates the leakage current when the device is in OFF state $\mathrm{V}_{\mathrm{dd}}$ is the supply voltage.

Leakage current is mainly depends on the following factors
a. Gate leakage
b. Sub threshold leakage
c. Reverse-biased junction leakage
d. Gate-induced drain leakage

From the all above factors it is found that the major components which affect the leakage power are sub-threshold leakage and gateleakage. The relationship of sub threshold leakage current is shown in (2)

$$
\begin{gathered}
I_{s u b}=I_{0} \exp \left[\left(V_{g s}-V_{t}\right) /\left(n V_{T}\right)\right]\left[-\exp \left(-V_{d s} / V_{T}\right)\right] \\
I_{0}=\mu_{e f f} C_{o x}(W / L) V_{T}^{2}
\end{gathered}
$$

Where,

$\mathrm{W}$ and $\mathrm{L}$ are channel length and width

Vt is the threshold voltage

$\mu$ eff is the electron/hole mobility

Cox is the gate oxide capacitance per unit area

$\mathrm{n}$ is the sub-threshold swing co-efficient

$\mathrm{V}_{\mathrm{T}}$ is the thermal voltage

$\mathrm{V}_{\mathrm{gs}}$ is the transistor gate to source voltage and

$\mathrm{V}_{\mathrm{ds}}$ is the drain to source voltage.

\section{PCS macro model}

Photo Catalytic Sensor is a MOSFET in which the gate terminal is floating and connected with chip in the form of reference electrode placed in an aqueous solution. ${ }^{17-19}$ The current-voltage relationship in active mode for PCS is same as that of MOSFET as given below in eq.4.

$$
I d s=C o x \mu W / L\left[\left(\mathrm{~V}_{\mathrm{gs}}-V_{t}\right) \mathrm{V}_{\mathrm{ds}}-1 / 2 \mathrm{~V}_{\mathrm{ds}}^{2}\right]
$$

Where,

Cox is the oxide capacitance per unit area,

$\mu$ is the electron mobility in the channel,

$\mathrm{W}$ and $\mathrm{L}$ are width and the length of the channel respectively.

In the above relationship shown in the equation 4 if all other parameters like $\beta, \mathrm{V}_{\mathrm{ds}}$ and $\mathrm{V}_{\mathrm{t}}$ are taken constant Ids is depends upon on $\mathrm{V}_{\mathrm{gs}}$ only. The relationship of threshold voltage with others parameters is given below in eq. 5 .

$$
\mathrm{V}_{\mathrm{t}}=\frac{\phi_{\mathrm{M}}-\phi_{\mathrm{si}}}{\mathrm{q}}-\frac{\mathrm{Q}_{\mathrm{ox}}+\mathrm{Q}_{\mathrm{ss}}+\mathrm{Q}_{\mathrm{B}}}{\mathrm{C}_{\mathrm{ox}}}+2 \phi_{\mathrm{f}}
$$

Where,

$$
\phi_{\mathrm{M}} \text { : Work function of gate metal. }
$$$$
\phi_{\mathrm{si}}: \text { Work function of silicon gate. }
$$

Qox: Oxide charge.

Qss: Interface charge carriers.

QB: Depletion charge carriers.

The fabrication steps for PCS and MOSFET are same due to which the threshold voltage equation of PCS looks a lot like with that of MOSFET. The equation included all necessary parameters as that is used in the formation of MOSFET equation. Although the concept of forming the equation is same but there are two additional following terms are also added.

(1) Constant reference electrode potential Eref.

(2) Interfacial potential which consists of chemical input parameter $(\Psi)$ which depends on concentration of $\mathrm{O} 2$ in the solution and surface dipole potential ( $\chi$ sol).

The above mentioned two parameters along with eq. 5 helps in the formation of threshold voltage equation for photo catalytic sensor and shown below in eq. 6 .

$$
V_{t h(P C S)}=E_{R e f}-\Psi_{\text {air }}+\chi^{\text {air }}+\frac{-\phi_{\mathrm{s}}}{\mathrm{q}}-\frac{\mathrm{Q}_{\mathrm{ox}}+\mathrm{Q}_{\mathrm{ss}}+\mathrm{Q}_{\mathrm{B}}}{\mathrm{C}_{\mathrm{ox}}}+2 \hat{o}_{f}
$$

As the characteristics of the MOSFET gate to source voltage the input gate to source voltage $\mathrm{Vgs}$ and the drain current $I_{d}$ is indorsed to vary with drain to source voltage while the reference voltage $\mathrm{E}_{\text {ref }}$ kept constant. The eq. 6 has been modified such that $\Psi$ air is taken as a function of $\mathrm{O}_{2}$ concentration in place of a function of $\mathrm{pH}$ as given by Grattarola et al.

On Comparing PCS with MOSFET keeping the concentration of $\mathrm{O} 2=1 \mathrm{mg} / \mathrm{l}$ constant, it is observed that the characteristic resembles with MOSFET keeping gate to source voltage constant as shown in Figure 2. At Vref. $=0 \mathrm{~V}$ it is observed that for different concentration levels of Oxygen, different Characteristics curves are obtained. From the above it is concluded that Photo Catalytic Sensor can be treated as MOSFET on the basis that the chemical input parameter $\Psi$ sol is a function of $\mathrm{O} 2$ ( $\Psi$ sol=f $($ Oxygen $)$ ).

The Modern analog building block used for designing current mode devices are Current Conveyor (CC-II) (Figure 3) introduced by Sedra and Smith. ${ }^{17-20} \mathrm{CC}$ has many application ranging from low power signal processing to implement of numerous analog processing applications. The Matrix relationship between input output current and voltage is shown below. The general CC can be expressed by the following input-output matrix relation:

$$
\left[\begin{array}{c}
I_{Y} \\
V_{X} \\
I_{Z}
\end{array}\right]=\left[\begin{array}{lll}
0 & a & 0 \\
1 & 0 & 0 \\
0 & b & 0
\end{array}\right]\left[\begin{array}{c}
V_{Y} \\
I_{X} \\
V_{Z}
\end{array}\right]
$$




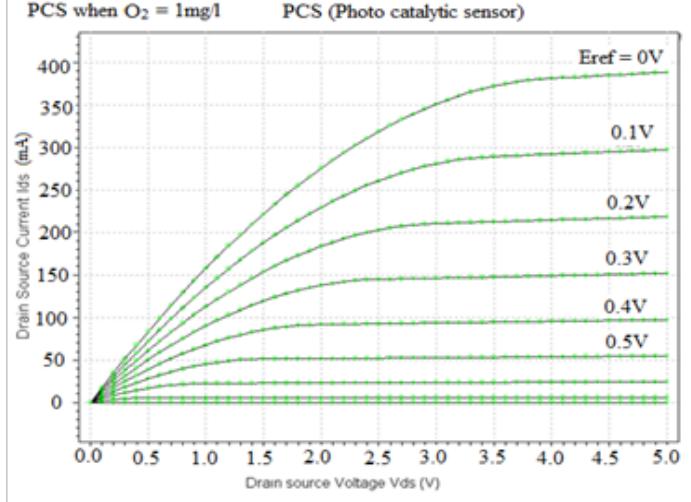

Figure 2 Simulation result of PCS as MOSFET.

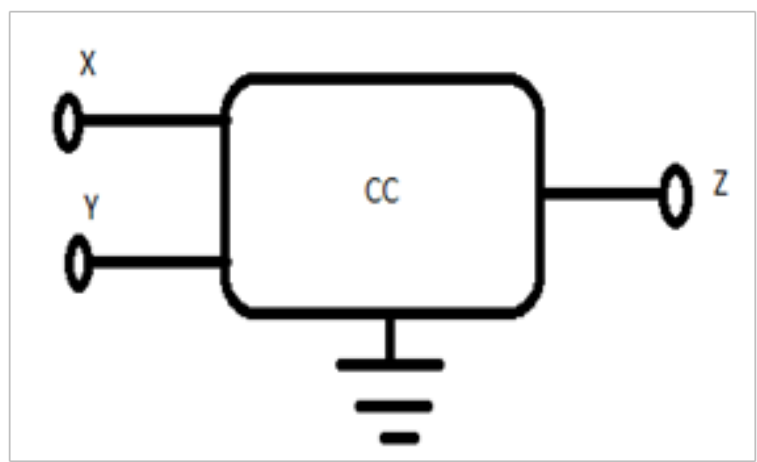

Figure 3 Block diagram current conveyor.

Different values of $\mathrm{a}$ and $\mathrm{b}$ leads to different functionality of the $\mathrm{CC}$ as follow

a. When value of $\mathrm{a}=1$, the first generation current conveyor $(\mathrm{CCI})$ is obtained.

b. At $\mathrm{a}=0$, we obtain the second generation current conveyor (commonly denoted CCII).

c. With $\mathrm{a}=-1$ we obtain the third generation current conveyor (commonly denoted CCIII)

d. Usually, $b= \pm 1$. The sign of the $b$ parameter determines the conveyor current transfer polarity.

e. For $\mathrm{b}=1$ indicates that the $\mathrm{CC}$ has a positive current transfer ratio and is denoted by $\mathrm{CCI}+, \mathrm{CCII}+$ or $\mathrm{CCIII}+$ while with negative $\mathrm{b}$ means that it has a negative current transfer ratio and is denoted by CCI-, CCII-or CCIII-

f. Form the all above discussed generation the second generation current conveyor (CCII) is most commonly used. An important attribute of current conveyor is its ability to convey current between two terminals ( $\mathrm{X}$ and $\mathrm{Z}$ ) at vastly different impedance levels as represented by the matrix.

\section{Pseusedo-NMOS}

logicPseudo-NMOS technique is based on ratioed logic in which a grounded PMOS is used as a Pull up. The NMOS driver circuit is used as pull-down network that realizes the logic function. The main advantage of this logic is it uses only $\mathrm{N}+1$ transistors as compared to $2 \mathrm{~N}$ transistors for CMOS logic. The CMOS logic has less load.
Capacitance on input signals, faster switching but have higher circuit density. In Pseudo-NMOS logic the high output voltage level for any gate is Vdd and the low output voltage level is not zero volts. ${ }^{20-25}$ The only main drawback of this logic is very high static power consumption which is not zero and exists because there is direct path between Vdd and ground. The size of PMOS device must be kept smaller than NMOS pull-down devices for making it low power. To increase the speed particularly when driving many other gates the PMOS transistor size has been increased. Therefore there is always a trade-off between the parameters noise margin, static power dissipation and propagation delay.

\section{Device description and analysis}

For the integrated sensor, the measurement circuit tracks the threshold voltage of the PCS which is varied as the concentration of $\mathrm{O} 2$ is varied. One of the solution to resolve the high power consumption problem by add-on sensor network is to integrate the sensor with electronics as a circuit component in an integrated circuit rather than as an add-on sensor whose output signals is further processed. In this research studies, the PCS is used as one of the input transistors in the differential stage of the current conveyor as shown in Figure 4. The function of the circuit is described as follows: In this circuit the PCS-Current Conveyor is configured as a voltage follower. In the voltage follower circuit the output voltage (Vo) is equal to the input voltage (Vin). This circuit is so sensitive that any difference in threshold voltages and bias currents between the two input transistors at the differential input stage will also appear at the output.

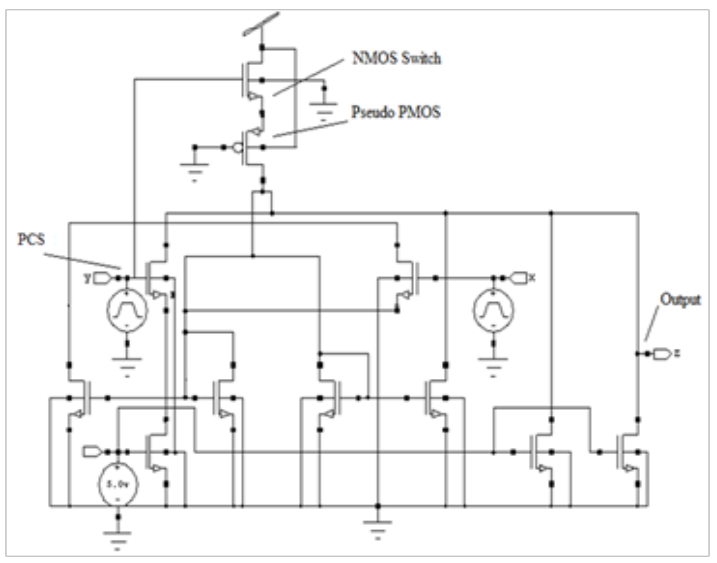

Figure 4 Circuit diagram of pseudo PMOS UVPCO.

The well-known distinct advantages of Pseudo PMOS like less load capacitance on input signals, faster switching due to fewer transistors and higher circuit density encourages us to implement this novel design. The only disadvantage of this logic is that the circuit is always on due to which there is significant static power dissipation which is a serious drawback. There are several methods to reduce the static power dissipation but there is no method so far technologically advanced to completely avoid this drawback. In this novel design the circuit has almost nil static power dissipation.

The circuit given below is designed using Pseudo PMOS technology in which gate of PMOS is grounded. There is one NMOS just above the grounded PMOS which act as a switch and ON only when the input is applied. As the circuit is $\mathrm{ON}$ along with the input signal hence there is no direct path from $\mathrm{V}_{\mathrm{dd}}$ to ground which prevent the circuit from the static power dissipation. 


\section{Timing analysis}

The analysis of a circuit output with respect to the time is called Transient analysis or timing analysis. The transient analysis of the PPUVPCO is observed on Tanner Tool. The device is found to be linear as the output is fairly linear with respect to input with the passage of time as shown in Figure 5 below

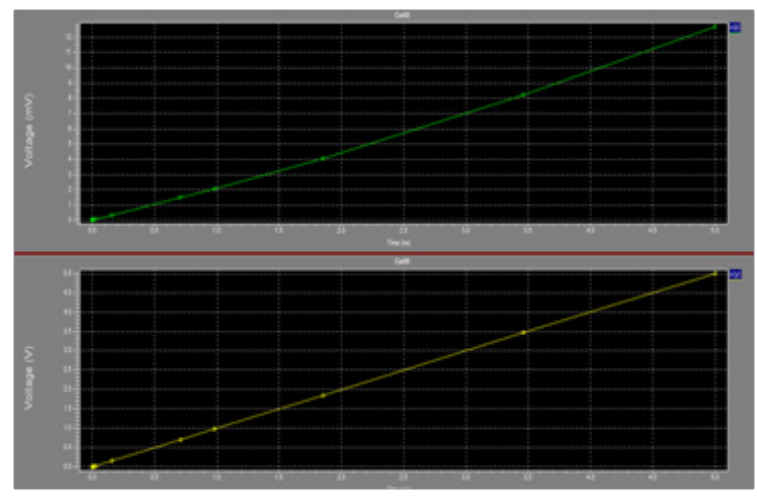

Figure 5 Transient analysis of device.

\section{Mathematical regression analysis}

The Regression statistics express the mathematical model of the device. It includes multiple R, R square and Adjusted R square, and Standard error value obtained during experiment. The various value obtained during the statistical analysis is shown in Table 1.

Table I Regression statistics

\begin{tabular}{lc}
\hline Regression & Statistics \\
\hline Multiple R & 0.998428255 \\
R Square & 0.99685898 \\
Adjusted R Square & 0.996662667 \\
Standard Error & 0.08121496 \\
Observations & 18 \\
\hline
\end{tabular}

The linear trend line between the readings of $\mathrm{V}_{\mathrm{y}}$ and $\mathrm{V}_{\mathrm{z}}$ is plotted and the coefficient of determination $\mathrm{R}^{2}$ is found to be $99.7 \%$ along with standard error of 0.081 . The coefficient of determination and standard error are shown in Figure 6. $\mathrm{R}^{2}$ (Coefficient of Determination) is useful because it gives the proportion of the fluctuation (variance) in other terms stability. This shows how one variable that is predictable from the other variable. That is why it is sometimes referred as predictive model. The coefficient of determination represents how well the regression line drawn from the data. If the regression line passes through each and every point on the scatter plot, this signifies that it would be easy to explain all the variations.

\section{Residual plot}

A residual plot is one of the statistical technique which is used to calculate how fit is regression model to your data. It should be random in nature the more the degree of randomness more the system is good to use. There should not be any recognizable pattern. The uncorrelated residuals models always give good value. Figure 7 shows random residual Plot for the device.

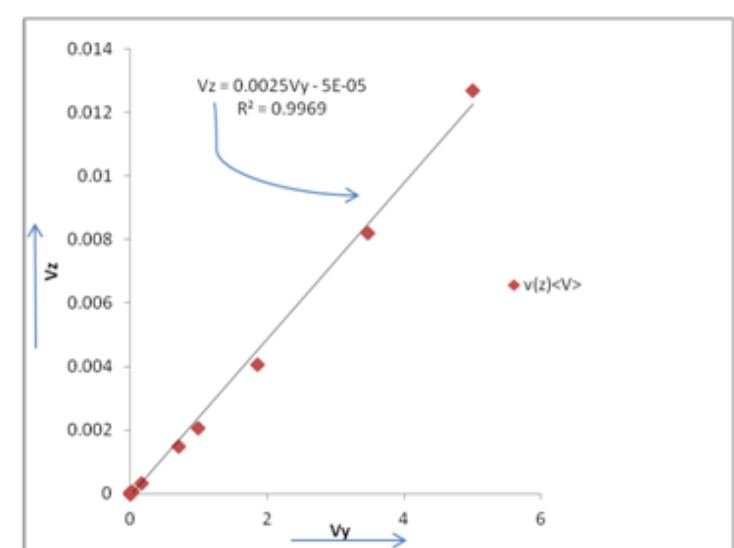

Figure 6 Trend line between $\mathrm{V}_{\mathrm{y}}$ and $\mathrm{V}_{\mathrm{z}}$ obtained from SPICE model readings with coefficient of determination.

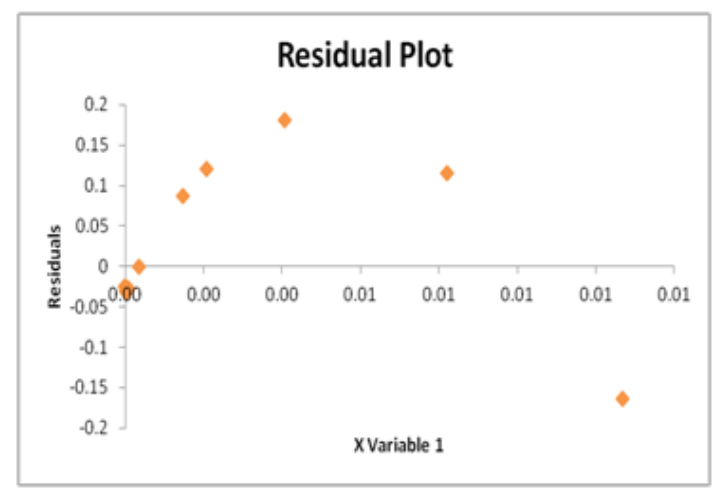

Figure 7 Residual plot for the device reading.

\section{Normal probability plot}

Normal Probability plot is the special case of probability plot which determines whether a given dataset can be uniformly distributed or not and also termed as a special case of the probability plot. If the points on this plot show a linear pattern, this indicates that the normal distribution is a good model for this data set. The figure 8 shows the normal distribution of the device dataset and it is clearly observed that the gradually linear increase in the values is a good model (Figure 8).

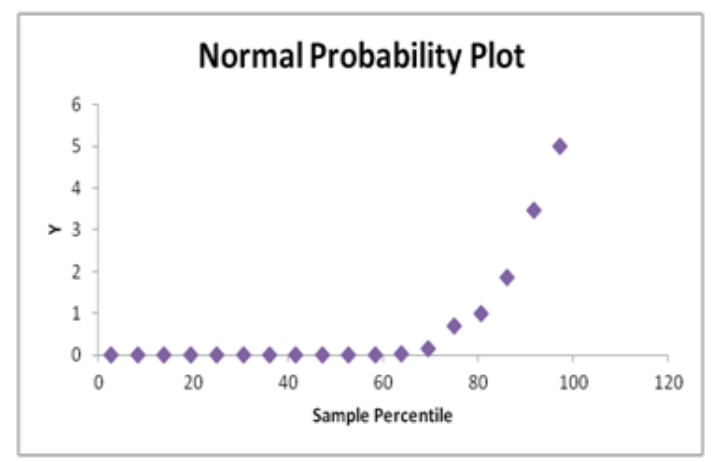

Figure 8 Normal probability plot.

\section{Result analysis}

The various results obtained are summarized in this section. On comparing new design with the existing design we arrive at following results 
1. Almost zero static power.

2. Only one capacitor is used in this technique.

3. Number of current sources deployed is zero in new device.

4. Number of n-MOS and p-MOS required: 8 and 1 .

5. No resistor is required in the new technique.

6. Only one Voltage source required.

Table 2 Analysis of PP-UVPCO devices

\begin{tabular}{ll}
\hline Parameters & PP-UVPCO \\
\hline Technology & Pseudo PMOS \\
Power supply(VDD, GND) & $5 \mathrm{~V}-0 \mathrm{~V}$ \\
No.of Mosfets & 9 \\
Capacitor & $\mathrm{I}$ \\
Current Source & 0 \\
NMOS & 8 \\
PMOS & $\mathrm{I}$ \\
Resistor & 0 \\
Voltage Source & $\mathrm{I}$ \\
Max power (Watts) & $3.000194 \mathrm{e}-002$ \\
Min power (Watts) & $0.000000 \mathrm{e}+000$ \\
Stability analysis & Closed loop stable \\
\hline
\end{tabular}

Volumetric efficiency is the measures of performance of function per unit volume. In today's scenario of electronics, miniaturization and low power circuit is a need for almost all circuits. From this table analysis, we can figure out that there is significant saving in terms of Components as compared to CMOS technology. Hence, to meet our goal a new circuit which meets all our requirements in terms of component saving i.e miniaturization and power efficiency is simulated and proposed in this circuit.

\section{Conclusion}

In this research studies, a new device employing Pseudo PMOSUVPCO is simulated. Pseudo PMOS Current Conveyor PPCC introduced as a basic building block in electronics circuits that provides a simplified approach to the design of linear analog systems. It consumes considerably low power and hence very useful for designing low power devices. The rate at which output changing with respect to the input i.e. slew rate also improved. A significant advantage of the proposed design is it's simple architecture and less number of component. Hence it is good for Air quality monitoring applications.

\section{Future work}

This study may be extended for further improvements in terms of power and size, besides the wiring and layout characteristics level.

\section{Acknowledgments}

None.

\section{Conflicts of interest}

The author declares there are no conflicts of interest.

\section{References}

1. Whig P, Ahmad SN. Simulation of linear dynamic macro model of photo catalytic sensor in SPICE. Compel-the Int J Comput Math Electric Electron Eng. 2014;33:611-629.

2. Whig P, Ahmad SN. Development of Economical ASIC For PCS For Water Quality Monitoring. JCSC. 2014;23(6).

3. Sze SM. Semiconductor Sensors. Wiley. New York; 1994.

4. Whig P, Ahmad SN. Performance analysis of various readout circuits for monitoring quality of water using analog integrated circuits. $I$. $J$. Intelligent Systems and Applications. 2012;11:91-98.

5. Kim YC, Sasaki S, Yano K, et al. Photocatalytic sensor for the determination of chemical oxygen demand using flow injection analysis. Analytica Chimica Acta. 2001;432(2):59-66.

6. Duffy JA. Bonding Energy Levels and Bands in Inorganic Solids. Wiley New York; 1990.

7. Massobrio G, Antognetti P. Semiconductor Device Modeling with SPICE New York; 1993.

8. Rodriguez-Villegas E. Low Power and Low Voltage Circuit Design with the FGMOS (IEE Circuits, Devices \& Systems). Institution of Engineering and Technology. 2006;20.

9. Peterson M, Turner J, Nozik A. Mechanistic studies of the photocatalytical behavior of $\mathrm{TiO} 2$ particles in photo electrochemical slurry and the relevance to photo detoxification reactions. Journal of Physical Chemistry. 1991;95:221-225.

10. Kahng D, SM Sze. A floating-gate and its application to memory devices. The Bell System Technical Journal. 1967;46(4):1288-1295.

11. Y Berg, Lande TS, Naess S. Low-voltage floating-gate current mirrors. 10th Annual IEEE International ASIC Conference and Exhibit. 1997;2124

12. Whig P, Ahmad SN. DVCC based Readout Circuitry for Water Quality Monitoring System. International Journal of Computer Applications. 2012;49(22):1-7.

13. Bergveld P. Development of an Ion-sensitive Solid-state Device for Neurophysiologic Measurements. IEEE Trans Biomedical Engineering. 1970;70-71.

14. Bergveld P. Thirty years of ISFETOLOGY what happened in the past 30 years and what may happen in the next 30 years. Sensors and Actuators B: Chemical. 2003;88(1):1-20.

15. Chang YH. Highly Sensitive $\mathrm{pH}$ Sensing Using an Indium $\mathrm{Ni}$ tride Ion-Sensitive Field-Effect Transistor. IEEE Sensors Journal. 2011;11(5):1157-1161

16. Firat Kacar. New CMOS Realization of Voltage Differencing Buffered Amplifier and Its Biquad Filter Applications. Radio Engineering. 2012;21(1):333-339.

17. Jamasb S, Collins SD, Smith RL. A physical model for thresh-old voltage instability in SI3N4 gate $\mathrm{H}+$ sensitive FETs. IEEE Tran Elec-tron Devices. 1998;45(6):1239-1245.

18. Pawan Whig, Syed Naseem Ahmad. A Novel Pseudo-PMOS Integrated ISFET Device for Water Quality Monitoring. Synopsys. TCAD Sentaurus device user's manual VG-2012.06.1. 
19. P Whig, Ahmad SN. On the Performance of ISFET-based Device for Water Quality Monitoring. International Journal of Communications, Network and System Sciences. 2011;4:709-719.

20. DM Wilson. Chemical sensors for portable handheld field in-struments. IEEE Sensors journal. 2001;1(4):256-274.

21. JT Kong. Method to improve Digital MOS Macro-Model Accuracy. IEEE Transactions on Computer-Aided Design of Integrated Circuits and Systems. 1995;14(7):868-888.

22. Buckley CA. Waste Water Reuse, The South African experience. Water Science and Technology. 2000;41(10):157-163.
23. Martinoia. Modelling non-ideal behaviour in Sensitive FETs with SPICE. Sensors and Actuators B: Chemical. 1992;7(1-3):561-564.

24. Waldner G, Roberto Gómez, Michael Neumann-Spallart. Using photo electrochemical measurements for distinguishing between direct and indirect hole transfer processes on anatase: Case of oxalic acid. Electrochimica Acta. 2006;52(7):2634-2639.

25. Nainani A, Yuan Z, Krishnamohan T, et al. Optimal design of III-V hetero structure MOSFETs. International Conference on Simulation of Semiconductor Processes and Devices. 2010;103-113. 